\title{
Oral Fibrolipoma: A Report of Two Cases
}

\author{
Maryam Jalili Sadrabad ${ }^{1}$, Shabnam Sohanian (iD) ${ }^{2}$ and Samira Behrad ${ }^{2, *}$ \\ ${ }^{1}$ Oral Medicine Department, Dental Faculty, Semnan University of Medical Sciences, Semnan, Iran \\ ${ }^{2}$ Oral and Maxillofacial Pathology Department, Dental School, Semnan University of Medical Sciences, Semnan, Iran \\ Corresponding author: Oral and Maxillofacial Pathology Department, Dental School, Semnan University of Medical Sciences, Semnan, Iran. Email: \\ samirabehrad84@gmail.com
}

Received 2019 September 22; Revised 2020 February 29; Accepted 2020 February 29.

\begin{abstract}
Introduction: Lipoma is a benign neoplasm of the fat tissue. Although, lipoma represents as the most common soft tissue neoplasm, lipomas of the oral cavity are very rare. This neoplasm contains $0.1-0.5 \%$ of all benign tumors of the oral cavity.

Case Presentation: We reported two cases of fibrolipomas; the first case was in a 36-year-old female as a swelling in retromolar pad area and the second one in a 71-year-old female as an exophytic mass in pterygomandibular raphe.

Conclusions: The novelty of these reported cases of fibrolipomas is that they have been occurred in infrequent area such as retro molar pad area and pterygo mandibular raphe. The lesions were removed by electro cutter with no recurrence at the follow up sessions. Although fibrolipoma is pretty rare in the oral cavity, there are several lesions can also have similar clinical features. Therefore, the histopathological examination is recommended for the clinicians in order to accomplish the final diagnosis.
\end{abstract}

Keywords: Fibrolipoma, Oral Buccal Lesion, Oral

\section{Introduction}

Lipoma represents the most common soft tissue neoplasm, which is $0.1 \%-0.5 \%$ of all benign tumors of the oral cavity $(1,2)$. The pathogenesis of lipoma is indistinctive and not associated with fat metabolism in the body (1). The buccal mucosa and buccal vestibule are the most common sites for oral lipomas and the tongue, the floor of the mouth and lips are the less common areas, respectively (1). This tumor occurs mostly in the 40-year-old or older patients and has no sex distribution (1). There are many microscopic variants of lipoma such as fibrolipoma, angiolipoma, spindle cell lipoma, pleomorphic lipoma, intramuscular lipoma, and sialolipoma; however, the most common type is fibrolipoma (1).

\section{Case Presentation}

In this study, two cases of fibrolipoma lesions in two women who were admitted at the Oral Medicine Department of Semnan Dental School are reported. The first patient was a 36-year-old woman who had noticed a swelling in her mouth since the year before. Her weight and systematic review were normal. The extraoral examination was not specific, but intraoral examination showed an exophytic mass with a soft consistency, pale pink and smooth, intact surface, approximately $1 \times 2 \mathrm{~cm}$ in dimensions. It was located in the retromolar pad area on the right side of the mandible (Figure 1). Due to the patient being asymptomatic during this period, she had not received treatment. After obtaining consent, the lesion was removed by electrocautery to prevent bleeding and fortunately, significant bleeding did not occur. The sterile compression pack was sufficient and there was no need for suturing.

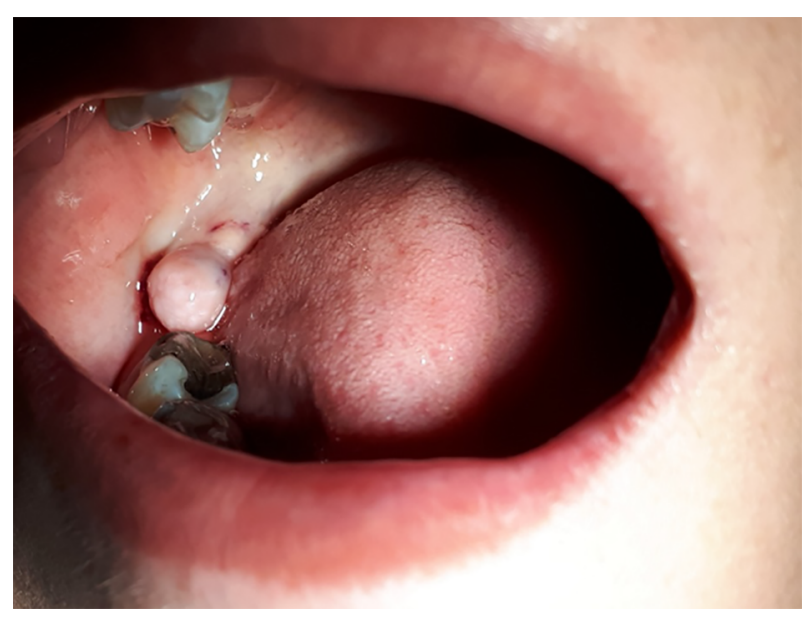

Figure 1. The first case of a 36-year-old woman with fibrolipoma preoperative

Copyright (c) 2020, Middle East Journal of Rehabilitation and Health Studies. This is an open-access article distributed under the terms of the Creative Common Attribution-NonCommercial 4.0 International License (http://creativecommons.org/licenses/by-nc/4.0/) which permits copy and redistribute the material just in noncommercial usages, provided the original work is properly cited. 
The second case was a 71-year-old woman who had already noticed the lesion for several years. Type 2 diabetes, hypertension, aspirin consumption, and overweightness were mentioned in history. The extraoral examination was normal, but a $3.5 \mathrm{~cm} \times 1.5 \mathrm{~cm}$ exophytic mass was seen intraorally, with a pink, smooth, intact surface located in the pterigomandibular raphe, which had a very soft consistency. Informed consent was obtained and the lesion removed by electrocautery, too. There was no significant bleeding, despite aspirin use, but the suture was closed to prevent probable bleeding.

In both cases after surgery, the specimen was sent to the Oral and Maxillofacial Pathology Department for histopathologic examination, and the result was fibrolipoma (Figure 2). Both patients were asked to refer to the clinic if the lesion was not properly removed or was recurrent, but they have not come back after 6 months.

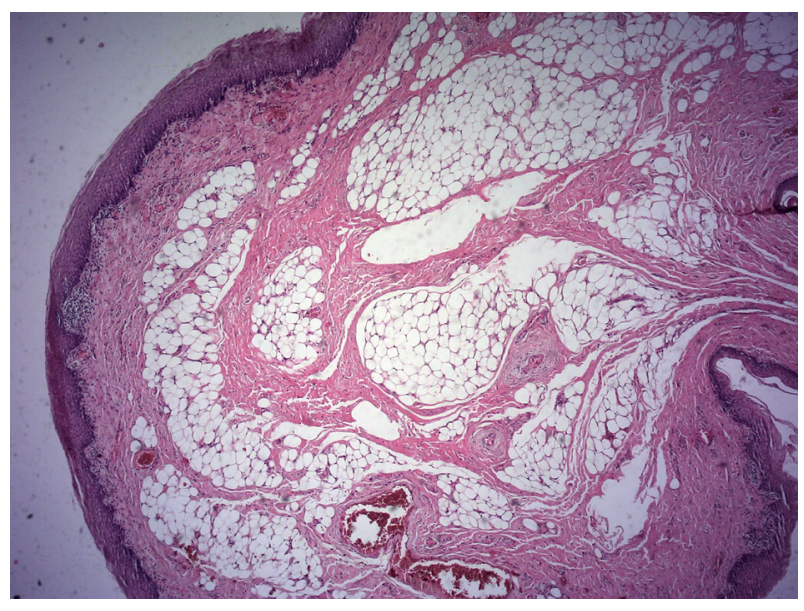

Figure 2. Buccal fibrolipoma $100 \times, \mathrm{H} \&$ E staining

\section{Discussion}

The lipoma is a benign soft tissue tumor, composed of mature lobulated adipocytes which are limited by a fibrous band (3). Clinically, alipoma presents as a solitary painless mass which is slow-growing and has a characteristic yellowish color with soft, doughy impression (4). The lesion is typically smooth and non-ulcerated at the surface unless it has been traumatized (4). This tumor is rare in the oral cavity and most seen in the buccal mucosa, lips, and tongue (1-5). Here, we reported two cases, one of them in the retromolar pad area and the other one located in pterygomandibular raphe. Lipomas often present in adults with a mean age of 60 years and are uncommon in children. Lipomas are frequently associated with chronic trauma (3,
4) family history, and diabetes (4). In this report, one of the patients was diabetic. Although numerous histologic variants are defined for lipoma, the most common microscopic variant of oral lipomas is the fibrolipoma (4). Fibrolipoma is characterized by a remarkable fibrous element intermixed with lobules of adipose tissue (5). There are several microscopic variants of lipoma including spindle cell lipoma, intramuscular or infiltrating lipoma, salivary gland lipoma, and myxoid lipoma $(3,6)$. The consistency of such tumors depends on the depth of the lesion and the quantity of fibrous tissue and it differs from soft to firm (5).

There are several lesions including lymphangioma, angioma, schwannoma, pyogenic granuloma, minor salivary gland neoplasms and liposarcoma, which are considered as the differential diagnosis of fibrolipoma (7). As a consequence of having a greater proliferative rate in fibrolipoma than other types of the lipomas, the accurate diagnosis of this variant is necessary in order to differentiate the lesion from atypical lipomatous tumor/well-differentiated liposarcoma (ALT/WDLS) $(7,8)$. Moreover, oral liposarcoma and its benign counterpart cannot be distinguished from each other at the clinical examination; hence, precise microscopic examination is obligatory. The differential diagnosis is most importantly based on the presence of lipoblasts in a variable amount in liposarcoma and also a lack of lobular architecture and areas of prominent fibrosis (8).

The treatment of fibrolipoma is entirely surgical $(9$, 10) but the use of diode laser surgery for oral fibrolipoma has been reported recently, which has several advantages in comparison with conventional blade surgery including the lack of bleeding, no requirement for suture and faster scar healing $(7,9,10)$. In these two cases, we used an electro cutter in order to remove the lesions. Although recurrence is rare, shcedulingfollow-up sessions is mandatory (3). Additionally, regressive tissue changes because of the thermal effect of the diode laser are frequently insignificant; therefore, allowing sufficient microscopic examination and accurate diagnosis (9).

\subsection{Conclusions}

We reported two cases of fibrolipoma that have occurred in infrequent areas such as the retromolar pad area and pterygomandibular raphe. The lesions were removed by an electro cutter with no recurrence at the follow-up sessions. Although fibrolipoma is pretty rare in the oral cavity, there are several lesions that can also have similar clinical features. Therefore, it is recommended that a clinician perform the histopathological examination for the final diagnosis. 


\section{Footnotes}

Authors' Contribution: Study concept and design: Maryam Jalili Sadrabad. Acquisition of data: Samira Behrad and Maryam Jalili Sadrabad. Analysis and interpretation of data: Shabnam Sohanian. Drafting of the manuscript: Maryam Jalili Sadrabad, Samira Behrad, and Shabnam Sohanian. Critical revision of the manuscript for important intellectual content: Shabnam Sohanian. Administrative, technical, and material support: Samira Behradand Shabnam Sohanian. Study supervision: Shabnam Sohanian

Conflict of Interests: There is no conflict of interest in this study.

Funding/Support: There is no funding or support.

Informed Consent: The informed consent was obtained.

\section{References}

1. de Visscher JG. Lipomas and fibrolipomas of the oral cavity. $J$ Maxillofac Surg. 1982;10(3):177-81. doi: 10.1016/s0301-0503(82)80036-2. [PubMed: 6957523].
2. Furlong MA, Fanburg-Smith JC, Childers EL. Lipoma of the oral and maxillofacial region: Site and subclassification of 125 cases. Oral Surg Oral Med Oral Pathol Oral Radiol Endod. 2004;98(4):441-50. doi: 10.1016/j.tripleo.2004.02.071. [PubMed: 15472660].

3. Le Gall J, Laurence S, Khalifa B, Boulagnon-Rombi C, Mauprivez C, Lefèvre B, et al. Intraoral fibrolipoma: Case report and review of literature. J Oral Med Oral Surg. 2018;24(3):129-32. doi:10.1051/mbcb/2018013.

4. Kumar P, Naraniya A. Intraoral fibro-lipoma: A rare histological variant. Indian J Oral Sci. 2012;3(1):39. doi: 10.4103/0976-6944.101675.

5. Iwase $\mathrm{M}$, Saida N, Tanaka Y. Fibrolipoma of the buccal mucosa: A case report and review of the literature. Case Rep Pathol. 2016;2016:5060964. doi: 10.1155/2016/5060964. [PubMed: 26885425]. [PubMed Central: PMC4739214].

6. Ganakalyan B, Kumar SD. Reviewing the entity: Retropharyngeal fibrolipoma and a rare case report. Iran J Otorhinolaryngol. 2015;27(83):469-74. [PubMed: 26788493]. [PubMed Central: PMC4709746].

7. Iaconetta G, Friscia M, Cecere A, Romano A, Orabona GD, Califano L. Rare fibrolipoma of the tongue: A case report. J Med Case Rep. 2015;9:177. doi: 10.1186/s13256-015-0653-1. [PubMed: 26293242]. [PubMed Central: PMC4546142].

8. Manjunatha BS, Pateel GS, Shah V. Oral fibrolipoma-a rare histological entity: Report of 3 cases and review of literature. J Dent (Tehran). 2010;7(4):226-31. [PubMed: 21998799]. [PubMed Central: PMC3184759].

9. Capodiferro S, Maiorano E, Scarpelli F, Favia G. Fibrolipoma of the lip treated by diode laser surgery: A case report. J Med Case Rep. 2008;2:301. doi: 10.1186/1752-1947-2-301. [PubMed: 18789134]. [PubMed Central: PMC2547114].

10. Mungul S, Maharaj S, Masege SD. Lingual fibrolipoma-A rare clinicopathological entity. S.Afr J Surg. 2017;55(2):36-8. 